GANIT J. Bangladesh Math. Soc. (ISSN 1606-3694) 37 (2017) 121-129

\title{
HEAT TRANSFER BEHAVIOUR INSIDE A SINUSOIDAL CAVITY USING WATER BASED $\mathrm{TIO}_{2}$ NANOfIUID
}

\author{
Goutam Saha \\ Department of Mathematics, University of Dhaka, Dhaka-1000, Bangladesh \\ E-mail: ranamath06@gmail.com
}

Received 05.06.2017

Accepted 02.11.2017

\begin{abstract}
A numerical investigation is carried out to observe the augmentation of heat transfer because of the presence of $\mathrm{TiO}_{2}$ nanofluid inside a sinusoidal cavity. In this study, upper and lower walls of the cavity are considered adiabatic, higher and lower temperature are maintained at left and right vertical walls respectively. Also, $2 \mathrm{D}$ contour of velocity and temperature with average heat transfer rate are presented and discussed. Our findings show that augmentation of heat transfer is feasible with the increase of concentrations of nanoparticles.
\end{abstract}

Keywords: Heat transfer; nanofluid; sinusoidal enclosure; Rayleigh number; concentrations.

\section{Introduction}

"In many practical applications, natural convection is the only feasible mode of cooling of the heat source. Further, the shape of the heat transfer surfaces influences the development of the boundary layer. Therefore, the investigation of thermal and fluid flow behaviors for different shapes of the heat transfer surfaces is necessary to ensure the efficient performance of the various heat transfer equipments" [1]. Moreover, many numerical investigations are performed on complex geometries of enclosures and also on sinusoidal cavities [2-14].

The physical geometry and the coordinate systems are shown in Fig. 1. It consists of a sinusoidal cavity of dimensions, $L \times L$. Also, left and right vertical walls are considered as hot and cold surfaces and the other horizontal walls are considered to be adiabatic. The range of Rayleigh numbers is considered to be $10^{3}$ to $10^{6}$ and nanoparticles concentration $\chi$ is varied from 0 to $15 \%$. [15]

We observe that no investigation has been done on the behaviours of heat transfer filled with nanofluid inside a sinusoidal cavity. The goal of this work is to examine the behaviour of heat transfer inside a sinusoidal cavity. Results of the velocity and temperature fields will be presented graphically and the average Nusselt number will be shown in tabular form. In addition, a new correlation will be presented for the calculation of $\overline{N u}$. 


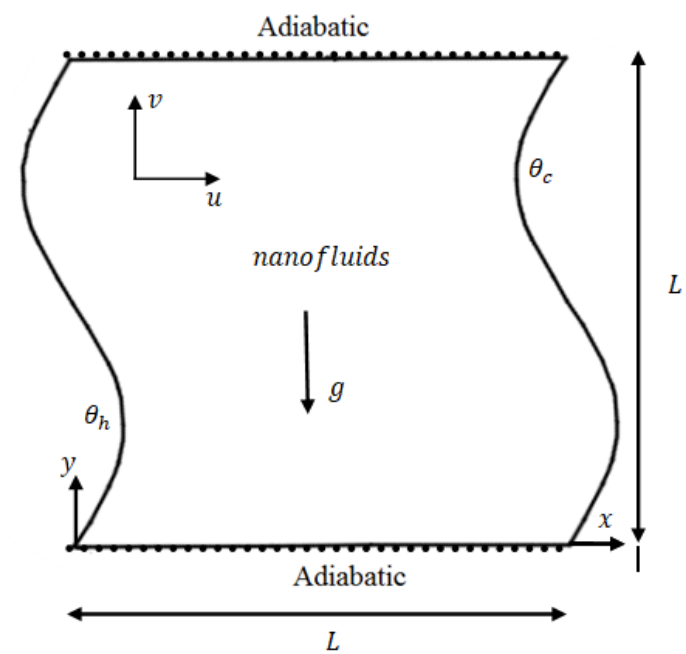

Fig. 1: Physical diagram

\section{Mathematical Model}

A steady state incompressible flow inside a sinusoidal cavity filled with water based $\mathrm{TiO}_{2}$ nanofluid is considered in the present investigation. Moreover, the well known Navier-Stokes equations with the Boussinesq approximation are presented below:

$$
\begin{aligned}
& \frac{\partial u_{1}}{\partial x_{1}}+\frac{\partial u_{2}}{\partial x_{2}}=0 \\
& u_{1} \frac{\partial u_{1}}{\partial x_{1}}+u_{2} \frac{\partial u_{1}}{\partial x_{2}}=-\frac{1}{\rho_{n f}} \frac{\partial p}{\partial x_{1}}+v_{n f}\left(\frac{\partial^{2} u_{1}}{\partial x_{1}{ }^{2}}+\frac{\partial^{2} u_{1}}{\partial x_{2}^{2}}\right) \\
& u_{1} \frac{\partial u_{2}}{\partial x_{1}}+u_{2} \frac{\partial u_{2}}{\partial x_{2}}=-\frac{1}{\rho_{n f}} \frac{\partial p}{\partial x_{2}}+v_{n f}\left(\frac{\partial^{2} u_{2}}{\partial x_{1}{ }^{2}}+\frac{\partial^{2} u_{2}}{\partial x_{2}{ }^{2}}\right)+(\rho \beta)_{n f} g\left(\theta-\theta_{c}\right) \\
& u_{1} \frac{\partial \theta}{\partial x_{1}}+u_{2} \frac{\partial \theta}{\partial x_{2}}=\alpha_{n f}\left(\frac{\partial^{2} \theta}{\partial x_{1}{ }^{2}}+\frac{\partial^{2} \theta}{\partial x_{2}^{2}}\right)
\end{aligned}
$$

where $u_{1}$ and $u_{2}$ indicates the velocities, $p$ is the pressure, $\theta$ is the temperature.

Nanofluids density, thermal diffusivity and heat capacitance are given as $[16,17,18]$

$$
\begin{aligned}
& \rho_{n f}=(1-\chi) \rho_{f}+\chi \rho_{s} \\
& \alpha_{n f}=\left(\frac{\kappa}{\rho c_{p}}\right)_{n f} \\
& \left(\rho c_{p}\right)_{n f}=(1-\chi)\left(\rho c_{p}\right)_{f}+\chi\left(\rho c_{p}\right)_{s}
\end{aligned}
$$

Nanofluids dynamic viscosity is given as (Brinkman [19]) 


$$
\mu_{n f}=\frac{\mu_{f}}{(1-\chi)^{2.5}}
$$

Also, nanofluids thermal conductivity, $\kappa_{n f}$ is given as (Maxwell [20])

$$
\frac{\kappa_{n f}}{\kappa_{f}}=\frac{\left(\kappa_{s}+2 k_{f}\right)-2 \chi\left(\kappa_{f}-k_{s}\right)}{\left(\kappa_{s}+2 k_{f}\right)-\chi\left(\kappa_{f}-k_{s}\right)}
$$

The boundary conditions are:

Vertical walls are hot and cold respectively.

Horizontal walls are adiabatic.

Let us consider the following non-dimensional variables:

$$
X_{1}=\frac{x_{1}}{L}, X_{2}=\frac{x_{2}}{L}, U_{1}=\frac{u_{1} L}{\alpha_{f}}, U_{2}=\frac{u_{2} L}{\alpha_{f}}, P=\frac{p L^{2}}{\rho_{n f} \alpha_{f}^{2}}, T=\frac{\theta-\theta_{c}}{\theta_{h}-\theta_{c}}
$$

Then, the dimensionless non-linear equations can be expressed as

$$
\begin{aligned}
& \frac{\partial U_{1}}{\partial X_{1}}+\frac{\partial U_{2}}{\partial X_{2}}=0 \\
& U_{1} \frac{\partial U_{1}}{\partial X_{1}}+U_{2} \frac{\partial U_{1}}{\partial X_{2}}=-\frac{\rho_{f}}{\rho_{n f}} \frac{\partial P}{\partial X_{1}}+\frac{\nu_{n f}}{\alpha_{f}}\left(\frac{\partial^{2} U_{1}}{\partial X_{1}{ }^{2}}+\frac{\partial^{2} U_{1}}{\partial X_{2}{ }^{2}}\right) \\
& U_{1} \frac{\partial U_{2}}{\partial X_{1}}+U_{2} \frac{\partial U_{2}}{\partial X_{2}}=-\frac{\rho_{f}}{\rho_{n f}} \frac{\partial P}{\partial X_{2}}+\frac{v_{n f}}{\alpha_{f}}\left(\frac{\partial^{2} U_{2}}{\partial X_{1}{ }^{2}}+\frac{\partial^{2} U_{2}}{\partial X_{2}{ }^{2}}\right)+\frac{(\rho \beta)_{n f}}{\rho_{n f} \beta_{f}} \operatorname{RaPr} T \\
& U_{1} \frac{\partial T}{\partial X_{1}}+U_{2} \frac{\partial T}{\partial X_{2}}=\frac{\alpha_{n f}}{\alpha_{f}}\left(\frac{\partial^{2} T}{\partial X_{1}{ }^{2}}+\frac{\partial^{2} T}{\partial X_{2}{ }^{2}}\right)
\end{aligned}
$$

The corresponding boundary conditions for the above problem are given by:

All walls: $U_{1}=U_{2}=0$, left side wall: $\mathrm{T}=1$ and right side wall: $\mathrm{T}=0$

Top and bottom walls: $\frac{\partial T}{\partial X_{2}}=0$

Also, the local and average Nusselt number are defined as

$$
N u=-\frac{k_{n f}}{k_{f}} \frac{\partial T}{\partial X_{1}} \quad \overline{N u}=\int_{\text {heated wall }} N u d X_{2}
$$

\section{Numerical Procedure}

It is really difficult to find the exact solution of non-dimensional PDEs with given boundary conditions. Therefore, an iterative scheme named FEM is considered in order to find the approximate solution of the given physical geometry. Moreover, six nodded triangular elements are considered throughout this investigation and TOL is considered as $10^{-8}$. Details of the finite element formulation are described in [1]. 


\section{Results and Discussion}

In this research, variation of velocity and temperature profiles with different $\mathrm{Ra}$ and $\chi$ inside a sinusoidal cavity are presented in Figs. 2-3. Besides, variation of average Nusselt number is also presented in Fig. 4. In this study, range of parameters such as $\mathrm{Ra}$ and $\chi$ are considered as $10^{3} \leq R a$ $\leq 10^{6}$ and $0 \leq \chi \leq 15 \%$ respectively. In addition, properties of different types of fluids are presented in Table 1.

Moreover, grid independence test is carried out for different $\mathrm{Ra}$ and lots of simulations have been performed in order to assess the grid distribution. It is found that non-uniform grid of 5044 elements is sufficient to capture the correct profiles of velocity and temperature distributions inside a sinusoidal cavity. Details of the results are given in Table 2. Also, a comparison has been done for different Ra with previous works as well as benchmark results as shown in Table 3. It is found that present solutions are in good agreement with the other published works.

Table 1: Properties of the water and $\mathrm{TiO}_{2}$ nanoparticles [21, 22]

\begin{tabular}{lll}
\hline $\operatorname{Pr}=6.2$ & Fluid Phase & Solid Phase \\
\hline Properties & Water, $300 \mathrm{~K}$ & $\mathrm{TiO}_{2}$ \\
$\mu\left(\mathrm{Ns} / \mathrm{m}^{2}\right)$ & $8.55 \times 10^{-4}$ & \\
$C_{p}(\mathrm{~J} / \mathrm{Kg} \mathrm{K})$ & 4179 & 686.2 \\
$\rho\left(\mathrm{Kg} / \mathrm{m}^{3}\right)$ & 997.1 & 4250 \\
$\kappa(\mathrm{W} / \mathrm{m} \mathrm{K})$ & 0.613 & 8.9538 \\
$\beta\left(\mathrm{K}^{-1}\right) \times 10^{-5}$ & 21 & 0.9 \\
$\alpha\left(\mathrm{m}^{2} / \mathrm{s}\right) \times 10^{-5}$ & & 0.31 \\
\hline
\end{tabular}

Table 2: Grid independency test $\left(\mathrm{Ra}=10^{6}\right.$ and $\left.\chi=0.01\right)$

\begin{tabular}{ccccccc}
\hline Elements & 2822 & 2948 & 3790 & 4206 & 4934 & 5044 \\
\hline$\overline{N u}$ & 5.58885 & 5.59914 & 5.50789 & 5.53774 & 5.54939 & 5.549931 \\
\hline
\end{tabular}

Table 3: Code validation works

\begin{tabular}{rlllll}
\hline Ra & Present & $\begin{array}{c}\text { Barakos and } \\
\text { Mitsoulis [23] }\end{array}$ & $\begin{array}{c}\text { Markatos and } \\
\text { Pericleous [24] }\end{array}$ & De Vahl Davis [25] & Fusegi et al. [26] \\
\hline $10^{3}$ & 1.118 & 1.114 & 1.108 & 1.118 & 1.105 \\
$10^{4}$ & 2.244 & 2.245 & 2.201 & 2.243 & 2.302 \\
$10^{5}$ & 4.515 & 4.510 & 4.430 & 4.519 & 4.646 \\
$10^{6}$ & 8.789 & 8.806 & 8.754 & 8.799 & 9.012 \\
\hline
\end{tabular}

Variation of streamline and isotherm profiles with different Rayleigh number for $\chi=0.15$ have been presented in Fig. 2. It is seen that flow moves along the clockwise pattern. It suggests that nanofluid particles inside the cavity moves up along the heated surface and then blocks at the adiabatic upper surface and then going down along the cooled surface and then moves towards the bottom adiabatic surface. 

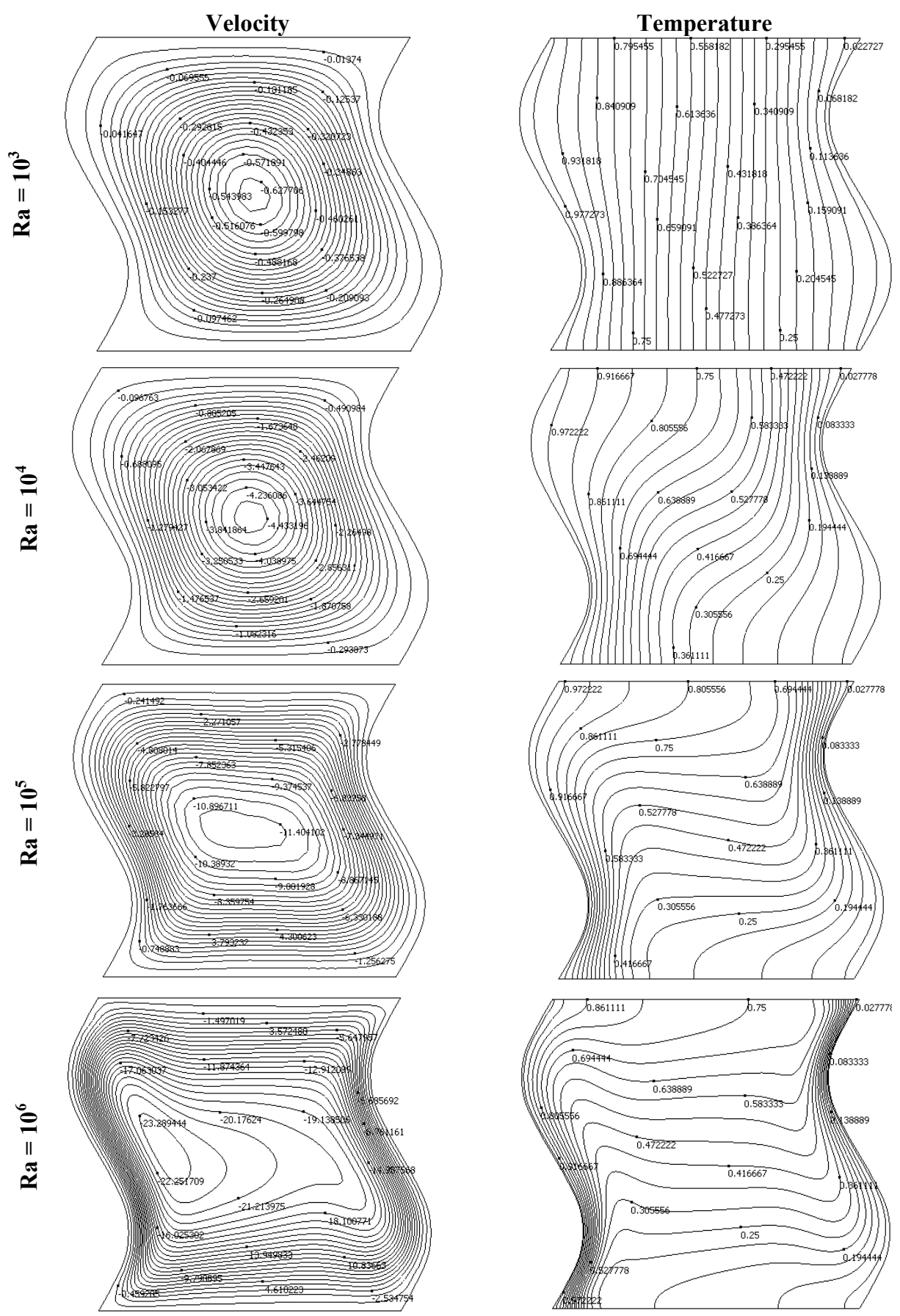

Fig. 2: Variation of velocity and temperature for $\chi=0.15$ 


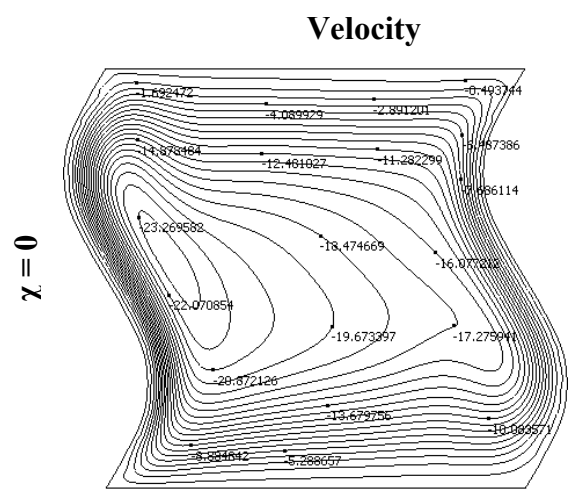

\section{Temperature}
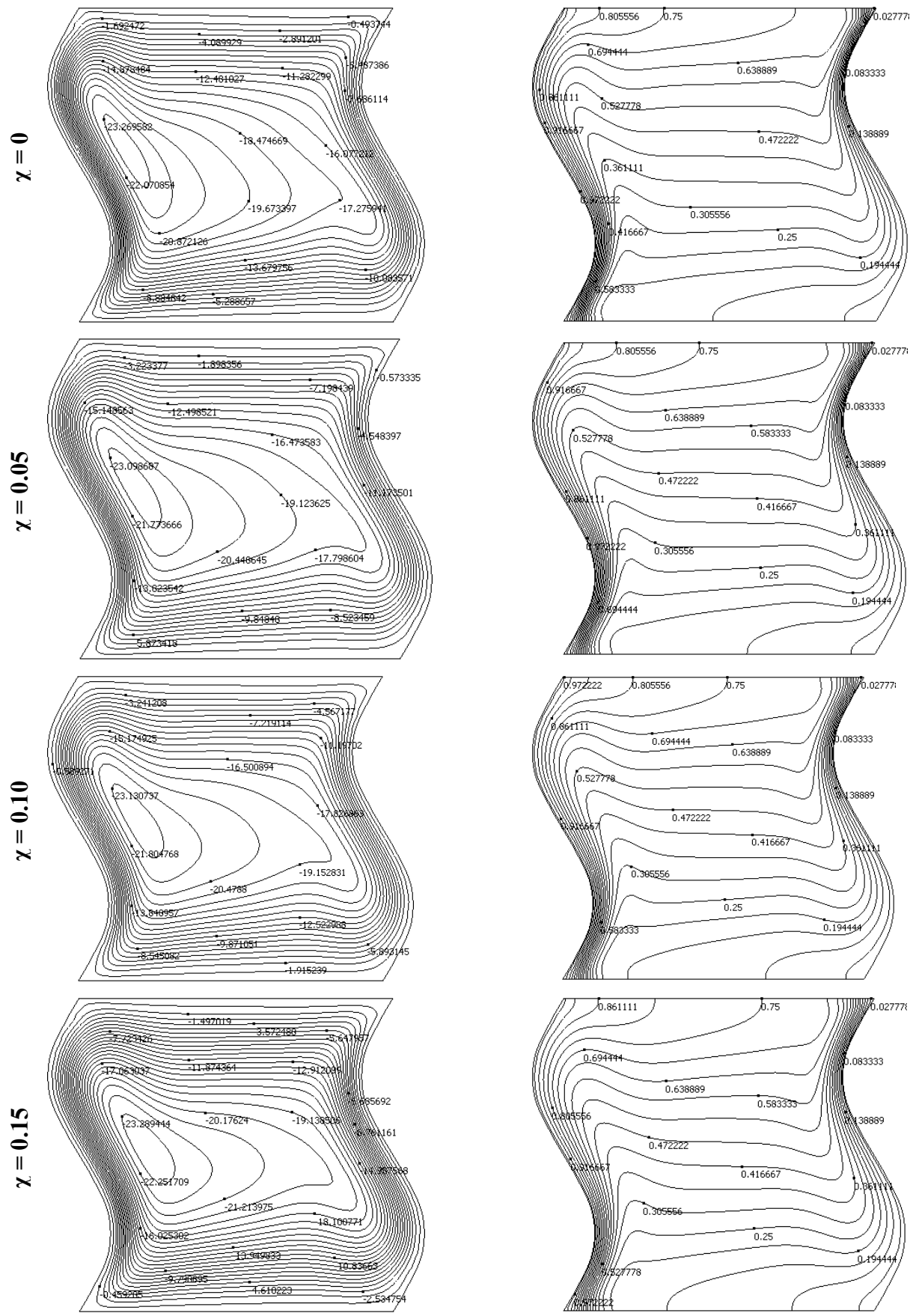

Fig. 3: Variation of velocity and temperature for various $\chi$ and $R a=10^{6}$ 
As shown in the Fig. 2, a single vortex is developed at low Ra. Also, the pattern of the central vortex is round in shape and it tends to become ellipsoidal with the increasing of Ra and in the end, central vortex moves near the left side wall and some deformations can be seen near the central zone for $R a=10^{6}$. It is to be noted that nanoparticles inclusion in the fluid affects the development of the vortex inside the cavity. Moreover, the strength of the flow circulation is superior with the increase of $\mathrm{Ra}$ when nanoparticles presence in the fluid taken is under consideration. It is due to the effect of nanoparticles concentrations as well as buoyancy force. Also, the temperature profile seems to be developed as a parallel layer along the vertical direction for low Ra. And, it tends to move towards the wall region with the increase of Ra and some horizontal layers are developed inside the centerline area of the cavity. This is also due to effect of buoyancy force.

Variation of streamline and isotherm profiles with different nanoparticles concentrations for $\mathrm{Ra}=$ $10^{6}$ have been presented in Fig. 3. It is found that elliptical shape centrally concentrated vortex is formed and developed towards the heated wall region for different concentrations. It is also found that in the absence of nanoparticles and for high $R a=10^{6}$, several vortex near the heated wall emerge as a leading attribute of the flow field. Also, the flow movement inside the cavity remains similar as discussed in Fig. 2. Moreover, as the nanoparticles concentrations increases, streamlines pattern seems to be squeezed at the central line position and dense near the boundaries. It is to be noted that maximum nanofluid velocity is also increased with the increase of concentrations. It is due to the higher nanoparticles concentration which helps to increase the thermal conductivity of nanofluids. This leads to the augmentation of energy which forces to speed up the flow. However, isotherm pattern seems to be similar to the previous results as shown in Fig. 2.

\section{Heat Transfer Correlation}

A new correlation has been proposed to calculate the average Nusselt number along the heated surface for $10^{3} \leq R a \leq 10^{6}$ and $0 \leq \chi \leq 0.15$. And the correlation is presented below:

$$
\overline{N u}=a\left(1+\chi^{n}\right) R a^{m}
$$

where $a=0.0783763, n=1.587369, m=0.3081744$ and $\mathrm{R}^{2}=99.9 \%$.

Table 4: Avg. Nusselt number, $\bar{N} \bar{u}$ for different $R a$ and $\chi$

\begin{tabular}{|c|c|c|c|c|c|c|c|c|}
\hline$P_{1}$ & $\chi=0$ & $1 \%$ & $2 \%$ & $3 \%$ & $4 \%$ & $5 \%$ & $6 \%$ & $7 \%$ \\
\hline Ka & \multicolumn{8}{|c|}{$\overline{\overline{N u}}$} \\
\hline $10^{3}$ & 0.649383 & 0.659271 & 0.669661 & 0.680562 & 0.702618 & 0.703909 & 0.716358 & 0.729323 \\
\hline $10^{4}$ & 1.287573 & 1.292638 & 1.297321 & 1.301619 & 1.361167 & 1.309037 & 1.312154 & 1.314874 \\
\hline $10^{5}$ & 2.694997 & 2.707888 & 2.720044 & 2.73147 & 2.853908 & 2.752137 & 2.761387 & 2.769921 \\
\hline $10^{6}$ & 5.448933 & 5.479941 & 5.50957 & 5.537847 & 5.780716 & 5.590425 & 5.614761 & 5.637811 \\
\hline$R a$ & \multicolumn{8}{|c|}{$\overline{N u}$} \\
\hline $10^{3}$ & 0.742802 & 0.756792 & 0.771291 & 0.786295 & 0.8018 & 0.817804 & 0.834303 & 0.851297 \\
\hline $10^{4}$ & 1.317204 & 1.319146 & 1.320714 & 1.321921 & 1.322788 & 1.323343 & 1.323618 & 1.323656 \\
\hline $10^{5}$ & 2.77775 & 2.784877 & 2.791314 & 2.797071 & 2.802156 & 2.806581 & 2.810357 & 2.813493 \\
\hline $10^{6}$ & 5.659589 & 5.680095 & 5.699341 & 5.717328 & 5.734055 & 5.749523 & 5.763728 & 5.776663 \\
\hline
\end{tabular}


Moreover, variation of average Nusselt number with different $R a$ has been presented in Table 4. It is seen that heat transfer rate increases with the increase of $R a$. It is evident that the values of $\overline{N u}$ increase as $R a$ increases from $10^{3}$ to $10^{6}$ for all values of nanoparticles concentrations.

\section{Conclusion}

Flow and heat transfer behaviours of nanofluid inside a 2D sinusoidal cavity have been presented and discussed for different $\mathrm{Ra}$ and $\chi$. Also, variation of streamlines and isotherms for a wide range of $\mathrm{Ra}$ and $\chi$ were presented graphically. Our findings reveal that heat transfer augmentation is possible using $\mathrm{TiO}_{2}$ nanofluid and such heat transfer rate is found higher compared with base fluid water. Moreover, nanoparticles concentrations have significant effect on the flow field, specially for high Ra.

\section{REFFERENCES}

[1] G. Saha, Finite Element Simulation of Magnetoconvection Inside a Sinusoidal Corrugated Enclosure with Discrete Isoflux Heating From Below, Int. Comm. Heat Mass Transfer 37(4) (2010) 393-400.

[2] S. Mahmud, P.K. Das, N. Hyder, A.K.M.S. Islam, Free convection in an enclosure with vertical wavy walls, Int. J. Thermal Sci. 41 (2002) 440-446.

[3] S. Mahmud, R.A. Fraser, Visualizing energy flows through energy streamlines and path lines, Int. J. Heat Mass Transfer 50 (2007) 3990-4002.

[4] Z. Sultana, N. Hyder Md., Non-darcy free convection inside a wavy enclosure, Int. Comm. Heat Mass Transfer 34 (2007) 136-146.

[5] P.K. Das, S. Mahmud, Numerical investigation of natural convection inside a wavy enclosure, Int. J. Thermal Sci. 42 (2003) 397-406.

[6] Y. Varol, H.F. Oztop, Free convection in a shallow wavy enclosure, Int. Comm. Heat Mass Transfer 33 (2006) 764-771.

[7] S. Abdelkader, R. Mebrouk, B. Abdellah and B. Khadidja, Natural convection in a horizontal wavy enclosure, J. Appl. Sci. 7 (2007) 334-341.

[8] K. Khanafer, B. Al-Azmi, A. Marafie, I. Pop, Non-Darcian effects on natural convection heat transfer in a wavy porous enclosure, Int. J. Heat Mass Transfer 52 (2009) 1887-1896.

[9] L. Yao, Natural convection along a vertical wavy surface, J. Heat Transfer 105 (1983) $465-468$.

[10] S. Saha, T. Sultana, G. Saha, M. Rahman, Effects of discrete isoflux heat source size and angle of inclination on natural convection heat transfer flow inside a sinusoidal corrugated enclosure, Int. Comm. Heat Mass Transfer 35 (2008) 1288-1296.

[11. M. Sheikholeslami, H.F. Oztop, MHD free convection of nanofluid in a cavity with sinusoidal walls byusing CVFEM, Chinese Journal of Physics (2017) (Accepted).

[12] W. Tang, M. Hatami, J. Zhou, D. Jing, Natural convection heat transfer in a nanofluid-filled cavity with doublesinusoidal wavy walls of various phase deviations, Int. J. Heat Mass Transfer 115 (2017) 430-440.

[13] M. Sheikholeslami, H.B. Rokni, Influence of EFD viscosity on nanofluid forced convection in a cavitywithsinusoidal wall, J. Molecular Liquids 232 (2017) 390-395.

[14] M. Sheikholeslami, M. Gorji-Bandpy, D.D. Ganji, .S. Soleimani, Natural convection heat transfer in a cavity with sinusoidal wall filled with $\mathrm{CuO}-$ water nanofluid in presence of magnetic field, J. Taiwan Institute Chemical Engineers 45 (2014) 40-49. 
[15] R.J. Issa, Effect of Nanoparticles Size and Concentration on Thermal and Rheological Properties of $\mathrm{AL}_{2} \mathrm{O}_{3}$-Water Nanofluids, Proceedings of the World Congress on Momentum, Heat and Mass Transfer (MHMT'16) Prague, Czech Republic - April 4-5, 2016 Paper No. ENFHT 101.

[16] G. Saha, M.C. Paul, Numerical analysis of heat transfer behaviour of water based $\mathrm{Al}_{2} \mathrm{O}_{3}$ and $\mathrm{TiO}_{2}$ nanofluids in a circular pipe under the turbulent flow condition, Int. Comm. Heat Mass Transfer, $\mathbf{5 6}$ (2014) 96-108.

[17] G. Saha, M.C. Paul, Heat transfer and entropy generation of turbulent forced convection flow of nanofluids in a heated pipe, Int. Comm. Heat Mass Transfer, 61 (2015) 26-36.

[18] G. Saha, M.C. Paul, Transition of Nanofluids Flow in an Inclined Heated Pipe, Int. Comm. Heat Mass Transfer, 82 (2017) p. 49-62.

[19] H.C. Brinkman, The viscosity of concentrated suspensions and solutions, J. Chem. Phys. 20 (1952) 571-581.

[20] J. Maxwell, A Treatise on Electricity and Magnetism, second ed. Oxford University Press, Cambridge, UK, 1904.

[21] E. Abu-Nada, Z. Masoud, A. Hijazi, Natural convection heat transfer enhancement in horizontal concentric annuli using nanofluids, Int. Comm. Heat Mass Transf. 35 (5) (2008) 657-665.

[22] E. Abu-Nada, A.J. Chamkha, Effect of nanofluid variable properties on natural convection in enclosures filled with a CuO-EG-Water nanofluid, Int. J. Thermal Sciences. 49 (2010) 2339-2352.

[23] G. Barakos, E. Mitsoulis, Natural convection flow in a square cavity revisited: laminar and turbulent models with wall functions, Int. J. Numer. Methods Fluids 18 (1994) 695-719.

[24] N.C. Markatos, K.A. Pericleous, Laminar and turbulent natural convection in an enclosed cavity, Int. J. Heat Mass Transfer 27 (1984) 772-775.

[25] G.D.V. Davis, Natural convection of air in a square cavity, a benchmark numerical solution, Int. J. Numer. Methods Fluids 3 (1962) 249-264.

[26] T. Fusegi, J.M. Hyun, K. Kuwahara, B. Farouk, A numerical study of three-dimensional natural convection in a di erentially heated cubical enclosure, Int. J. Heat Mass Transfer 34 (1991) 15431557. 